\title{
Autopistas, Ferrocarriles y Bifaces: ¿nuevas perspectivas para la investigación del Paleolítico en los ríos Manzanares y Jarama? *
}

\author{
Susana Rubio jara, Joaquín Panera Gallego \\ Y JuAn ANTONIO MaRToS RoMERo
}

\begin{abstract}
RESUMEN
ABSTRACT

Tal vez la gran aportación tras un siglo

After near a century of investigations de investigación en los valles Jarama y in the Jarama and Manzanares

Manzanares haya sido dejar valleys maybe the main conclusion is constancia de su gran potencial arqueológico. En la actualidad debido its valuable Archaeological heritage. At present due to the serious and

al grave $y$ acelerado deterioro que sufre la zona es necesario un esfuerzo para encontrar un marco adecuado en el que la investigación del Paleolítico pueda desarrollarse. accelerated damage that we can see in this area, it's neccesary to find an appropriate framework where the investigation of the Paleolithic can grow.

\section{PALABRAS CLAVES}

Paleolítico inferior y medio; patrimonio

KEY WORDS

Lower and Middle Paleolithic; arqueológico; legislación
\end{abstract}

«En las excavaciones que se hacen junto al puente de Toledo, a mano izquierda, saliendo por la puerta del mismo nombre, para la formación de aquellos caminos, se han encontrado à fines de Septiembre, à vara y media de profundidad, en un terreno duro, gredoso y virgen, algunos huesos de Elefante; es à saber: las puntas de una de las muelas grandes, la choquezuela de una rodilla y otros diferentes fragmentos». Gaceta de Madrid, 29 noviembre de 1778.

Mariano de la Paz Graells, Fauna Mastológica Ibérica (1897)

* Este artículo fue remitido el 3 de febrero de 2000.

** Departamento de Prehistoria e Historia Antigua de la UNED Senda del Rey s/n, 28040 Madrid. 
La finalidad del presente artículo es poner en valor el patrimonio arqueológico de Madrid, y en nuestro caso el perteneciente a las primeras ocupaciones humanas de los valles Jarama y Manzanares, en un momento en el que se encuentra gravemente amenazado por la creación de nuevas vías de comunicación. Los arqueólogos debemos conocer el marco legislativo para poder avanzar en la protección, investigación y difusión de dicho patrimonio.

Transcurridos más de dos siglos desde que aparecieran los primeros restos paleontológicos y arqueológicos en el valle del Manzanares, contamos por primera vez con un marco legal adecuado que protege explícitamente el patrimonio cultural. Primero con lo dispuesto en la ley 16/85 de Patrimonio Histórico Español, y posteriormente con la Ley de Patrimonio Histórico de la Comunidad de Madrid (Ley 10/1998, de 9 de julio, BOCM del 16 de julio). Además nuestro área de interés se encuentra extraordinariamente afectada por la creación del Parque Regional en torno a los ejes de los cursos bajos del Jarama-Manzanares (Ley 6/94 de 28 de junio, BOCM del 12 de julio de 1994), concretado por el Plan de Ordenación de los Recursos Naturales del 11 de febrero de 1999 (Decreto 27/1999, BOCM del 3 de marzo).

Sin entrar a analizar pormenorizadamente el contenido de estos textos legales, para lo que se pueden consultar las publicaciones de Querol y Martínez Díaz (1996a; 1996b;1998), es oportuno destacar los aspectos más relevantes para la investigación del paleolítico en el Jarama y Manzanares.

\section{CON LA LEY EN LA MANO}

La ley autonómica, como la de Patrimonio Histórico Español, establece tres grados de protección (Art. 8): Bienes de Interés Cultural (BIC), bienes incluidos en el inventario, y bienes que no han sido objeto de inventario $O$ declaración, siendo los más exigentes para los BIC. Éstos se catalogan en ocho tipos dentro de los que destacamos (Art. 9.2): Zonas Arqueológicas, "lugar o paraje natural en donde existan bienes muebles o inmuebles 0 restos de intervención humaná, susceptibles de ser estudiados con metodología arqueológica, tanto si se encuentran en la superficie como si se encuentra en el subsuelo, bajo las aguas o en construcciones emergentes" y Zonas Paleontológicas, "lugar donde hay vestigios fosilizados que constituyen una unidad coherente y con entidad propia".

El tramo final del Manzanares y el área en torno a su confluencia con el Jarama están incoados como Bien de Interés Cultural por el Consejo de 
Gobierno de la Comunidad de Madrid. La ley madrileña además de señalar el deber de conservar los bienes culturales tanto por los particulares como por los poderes públicos establece dos hechos relevantes a nuestro juicio: «En las actuaciones que afecten a un lugar arqueológico declarado BIC se requerirá la evaluación de impacto ambiental previo informe de la Dirección General de Patrimonio Cultural» (Art. 32.1.g.); «no se permitirá ningún tipo de edificación hasta que no se realice un estudio de la incidencia que las obras pueden tener en los restos arqueológicos, elaborado por un profesional competente en la materia. Previamente a la concesión de la licencia será precisa la autorización de la Dirección General de Patrimonio Cultural. La citada autorización deberá incluir como condición para la ejecución de las obras, la realización y la ejecución de un proyecto arqueológico...» (Art. 40.2).

Así mismo aunque no se trate de una zona declarada BIC «Si durante la ejecución de cualquier tipo de obra o movimientos de tierra, se hallaren restos $u$ objetos con valor arqueológico o paleontológico, el promotor o la dirección facultativa de la obra paralizarán inmediatamente los trabajos, tomarán las medidas adecuadas para la protección de los restos y comunicarán su descubrimiento en el plazo de 48 horas...» (Art. 45.1).

En junio de 1994 la Asamblea de Madrid aprobó por unanimidad la creación del «Parque Regional en torno a los ejes de los cursos bajos de los ríos Jarama y Manzanares" debido a la creciente demanda social que venía exigiendo la protección y recuperación de un espacio de alto valor ecológico y arqueológico: "...considerando como enclave central la confluencia de los ríos Jarama y Manzanares, se extiende un área cuya calidad ambiental está definida por los contrastes que suponen la coexistencia de zonas de alto valor ecológico, paleontológico y arqueológico y la degradación producida por la actividad industrial, la inadecuada explotación de los recursos y factores derivados de su carácter periurbano" (Preámbulo de la Ley 6/94).

El Parque se vertebra principalmente por los ríos Jarama y Manzanares y en menor medida por el Henares. Ocupa una amplia extensión de terreno, unos $300 \mathrm{~km}^{2}$, que incluye a 16 municipios entre San Fernando de Henares y Aranjuez. La Ley que lo regula tiene como primer objetivo "proteger la gea, fauna, flora, agua, atmósfera y paisaje, así como los restos arqueológicos y paleontológicos de todo el conjunto de los ecosistemas del ámbito ordenado..." (Art. 1.a).

El territorio del Parque se divide en Zonas con distinto nivel de protección y usos autorizados, que va disminuyendo desde las Zonas A a las F: 
- Zonas $A$ «de reserva integral».

- Zonas $B$ «De reserva natural».

- Zonas $C$ «Degradadas a regenerar».

- Zonas D «Explotación ordenada de los recursos naturales».

- Zonas $E$ «Destino agrario, forestal, educacional y/o equipamientos ambientales y/o usos especiales".

- Zonas $F$ «De protección».

Uno de los elementos que más directamente amenazan el registro arqueológico y paleontológico del Manzanares y Jarama son las canteras que han provisto de áridos a Madrid desde el siglo pasado. En las zonas $A, B, C$ y $E$ la Ley 6/9 establece la prohibición de concesión de nuevas autorizaciones de extracción de áridos (Arts. 27 a 31), y se remite a las graveras que explotaban áridos en aquel momento en dichas áreas a las acciones que determinara el Plan de Ordenación de los Recursos Naturales, el cual debería estar aprobado en el plazo máximo de un año (Art. 10).

El controvertido Plan de Ordenación de los Recursos Naturales no fue aprobado hasta el 11 de febrero de 1999, es decir con un retraso de casi cuatro años según lo señalado en la Ley 6/94. El Plan establece entre los objetivos de las Normas relativas a la ordenación de los recursos naturales "proteger el patrimonio cultural de cualquier actuación que pueda suponer un menoscabo o deterioro de sus valores» y «Potenciar la difusión del conocimiento del mismo» (Art. 10.6.1.), con las siguientes directrices: «Se tendrá en cuenta la riqueza del patrimonio cultural de los yacimientos arqueológicos, paleontológicos (...) en las actividades que se realicen en el ámbito del PORN"; "Se fomentarán los trabajos de investigación, protección, estabilización, restauración y difusión de los bienes de interés histórico artístico y cultural del área de ordenación y de su zona de influencia». También consideramos oportuno destacar entre su Normativa que: «Previamente a la concesión de cualquier autorización para la realización de actividades que supongan la alteración del suelo, se requiere por parte de la Consejería de Medio Ambiente, certificado emitido por la Consejería de Educación y Cultura de haberse realizado los trabajos de prospección arqueológica, así como los resultados y conclusiones obtenidos en los mismos"; "Una vez realizados los trabajos de prospección arqueológica y paleontológica, independientemente de los resultados obtenidos durante el desarrollo de la actividad, se tendrá que realizar una supervisión arqueológica y paleontológica por parte de la Consejería de Educación y Cultura..." (Art. 10.6.3.c y d).

Respecto a las actividades extractivas, considera compatible el aprovechamiento de los recursos mineros en las zonas $D$ y $F$, mientras que 
las canteras que se encuentren en las restantes zonas deberán concluir sus actividades en el plazo máximo que contemplaba la ley, cinco años, pese a que un borrador redactado en octubre de 1997 estableciera que no se prorrogaran las concesiones de explotación de gravas en las zonas $A, B, C$ y $E$, y tampoco se permitiera la explotación en las zonas $\mathrm{D}$ y $\mathrm{F}$.

Una vez vistas las herramientas legales que tenemos a nuestra disposición vamos a describir la problemätica por la que atraviesan las cuencas del Jarama y Manzanares.

\section{DESARROLLO DE INFRAESTRUCTURAS}

En la actualidad la Comunidad de Madrid y su entorno están sufriendo una profunda transformación en su configuración urbana, paisajística y red viaria, que afecta extraordinariamente a nuestra área de investigación por diversos motivos: el acelerado crecimiento del extrarradio de Madrid y de los núcleos urbanos periféricos; la implantación de nuevas vías de comunicación que afectan directamente al cauce del Jarama; y la creación de nuevos enclaves ecológico-culturales. Los dos primeros hechos implican, además, que la demanda de áridos se haya multiplicado considerablemente en los últimos años.

La creciente demanda de áridos ha provocado que se reabran antiguas canteras y que se creen otras nuevas, no siempre con las licencias administrativas pertinentes. Las graveras explotan de forma masiva y agresiva los depósitos fluviales del Jarama y en menor medida del Manzanares, vaciando amplias zonas, de hasta varios $\mathrm{km}^{2}$, en breves períodos de tiempo. Procediendo a continuación al relleno artificial de estas oquedades, y en ocasiones restaurando el paisaje mediante repoblación vegetal en cumplimiento de la normativa vigente al respecto.

Se están acometiendo importantes obras viarias que afectan a los depósitos del Jarama, como son la M-45 o el AVE Madrid-Barcelona. En un futuro próximo se llevarán a cabo otras de mayor incidencia si cabe para este río como son la $M-50$ o la R-3 (Fig. 1), incluso la M-60 y $M-70$ que ya están diseñados.

La M-45, ya construida, la M-50 y el trazado del AVE atraviesan el cauce y las terrazas del Jarama a la altura de Mejorada del Campo. El AVE según asociaciones ecologistas originará la destrucción del bosque de ribera del río Henares y la segregación de toda la zona norte del 


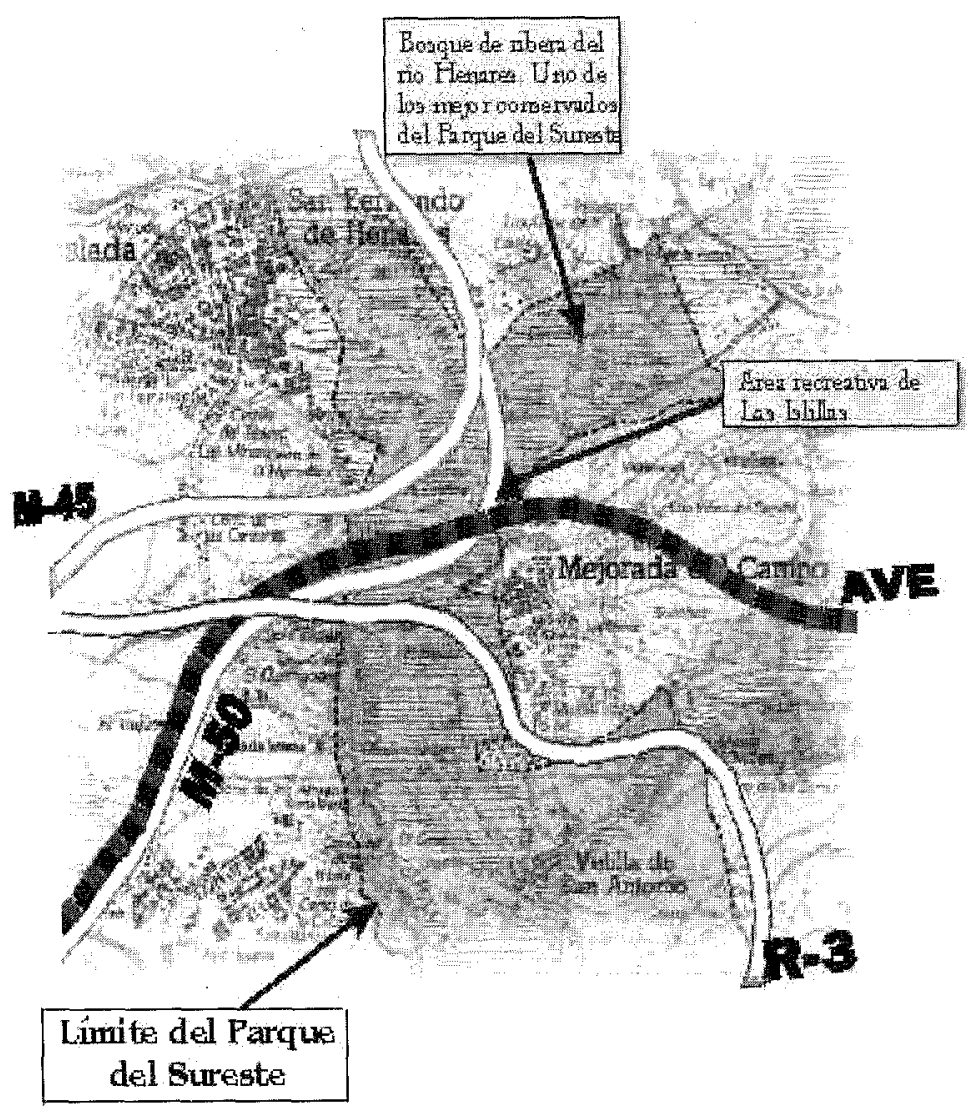

Fig. 1. Trazado de las diferentes autopistas y AVE a su paso por el Jarama (según la revista «El Taray», num. 11, octubre de 1999).

Parque (desde Velilla de San Antonio hasta San Fernando). Debemos considerar que necesita curvas muy amplias (varios $\mathrm{Km}$. de radio) y pendientes muy reducidas (menores del 2 ' $5 \%$ ), que obliga a hacer grandes desmontes de terreno (de hasta $160 \mathrm{~m}$. de anchura), altísimos viaductos, etc.

El Ministerio de Fomento a principios de septiembre de 1999 adjudicó la construcción de la autopista R-3, que pretende crear un nuevo acceso a Madrid desde Levante. Atravesará Rivas-Vaciamadrid y cruzará el Jarama para recorrer Mejorada del Campo y Velilla de San Antonio, continuará por las terrazas del Jarama y subirá por las cuestas del arroyo Cacera, hasta enlazar con la A-3 en Arganda del Rey. 
Autopistas, Ferrocarriles y Bifaces ¿nuevas perspectivas para la investigación...

Por otro lado en el tramo final del Manzanares se está proyectando la creación de un gran parque que se extiende a lo largo de una franja de 10 $\mathrm{km}$. y abarca más de 600 hectáreas.

Ante esta situación la investigación de las ocupaciones humanas pleistocenas en la Cuenca de Madrid atraviesa por momentos críticos y al mismo tiempo decisivos. Probablemente nos encontramos ante una de las últimas oportunidades para acceder al amplio potencial arqueológico que encierra la zona. Pero si bien todo lo mencionado anteriormente impedirá en un futuro a medio plazo la investigación en este área, como en la actualidad sucede con el tramo del Manzanares que discurre por el núcleo urbano de Madrid donde se ubican relevantes yacimientos como el de San Isidro, pone a nuestro alcance la posibilidad de prospectar centenares de metros de secciones de los depósitos del Jarama y Manzanares donde ubicar yacimientos paleolíticos en estratigrafía, que en otro contexto sería imposible inspeccionar.

\section{UN PASO MÁS HACIA EL CONOCIMIENTO DE LAS OCUPACIONES HUMANAS PLEISTOCENAS EN EL ÁREA DE MADRID}

Si bien las numerosas publicaciones existentes acerca del paleolítico en el Jarama y Manzanares - Vernuil y Lartet (1863), Prado (1864), Pérez de Barrados (1923), Obermaier (1930), Santonja et alii (1980), Rus (1983) y Baena (1992) - pudieran hacer pensar en una continuidad de la investigación, en nuestra opinión la realidad parece ser bien distinta. Muy al contrario, lo que habría que destacar es precisamente una falta de continuidad de los proyectos de investigación, limitándose ésta a intervenciones muy puntuales, llevadas a cabo por no especialistas en algunas ocasiones, y con una temporalidad entre ellas de más de dos décadas. Esta situación - después de más de 100 años desde los primeros hallazgos-demanda la existencia de un proyecto de investigación que con independencia de las propuestas que plantee permita analizar de forma coherente el estudio del paleolítico en estos valles.

Con esta intención reunimos un equipo interdisciplinar compuesto por geólogos, arqueólogos especialistas en Paleolítico inferior y paleontólogos que comenzó a trabajar en 1996. Desde entonces los esfuerzos se han concretado en dos proyectos de investigación. El primero de ellos, concedido por la Dirección General de Patrimonio Cultural de la C.A.M. (referencia 09/059643.2/98), se llevó a cabo durante los meses de octubre y noviembre de 1998, y consistía en un permiso de prospección y el correspondiente estudio de los materiales arqueológicos recogidos en las te- 
rrazas del Jarama en los términos municipales de Mejorada del Campo, Velilla de San Antonio, Arganda del Rey y Rivas Vaciamadrid (Fig. 2). El segundo proyecto bajo el título "Puesta en valor de las primeras ocupaciones humanas de la cuenca de Madrid" ha sido recientemente concedido por la Dirección General de Investigación de la C.A.M. para el año 2000 (N. ${ }^{\circ}$ Expediente: 06/0001/99) y pretende, entre otros objetivos, dotar de un marco cronológico válido los eventos culturales del Pleistoceno de la Cuenca de Madrid.

Al comenzar nuestra intervención en los valles Jarama-Manzanares observamos dos hechos. Primero, que a pesar de la larga trayectoria investigadora en el área aún faltan datos concluyentes sobre la paleoecología de las ocupaciones homínidas pleistocenas en Madrid, y segundo, que la secuencia estratigráfica de estos valles aunque esbozada no está concluida. Nos planteamos elaborar una secuencia estratigráfica de las ocupaciones, para lo que es necesario ubicar conjuntos industriales en sus distintas terrazas, y documentar yacimientos paleolíticos en contextos sedimentarios de baja energía que aporten información acerca del comportamiento de los homínidos del Pleistoceno medio. Ante la escasez de cronologías fiables para estos depósitos, juzgamos que la existencia en estos momentos de mejores métodos de datación haría interesante su aplicación.

Tras una exhaustiva revisión bibliográfica, acometimos la consulta de la Carta Arqueológica de los términos municipales de Velilla de San Antonio, Mejorada del Campo, Arganda del Rey y Rivas Vaciamadrid, con una aportación de 87 yacimientos que distribuidos por términos municipales se reparten del siguiente modo: para Velilla 11 conjuntos, en Mejorada 23, en Arganda 12 y en Rivas 41. Estos hallazgos son calificados culturalmente con categorías tipológicas que van desde Achelenses de tradición musteriense a Musterienses tipo Quina o Charetienses demasiado estrictas para ser aplicadas a conjuntos industriales en superficie.

El área mejor conocida arqueológica y geológicamente es la denominada región de Arganda, gracias a los trabajos llevados a cabo por Manuel Santonja (Santonja et alii, 1980; Santonja, 1980; Santonja y Querol, 1977) y por Alfredo Pérez González $(1971 ; 1994)$ y donde consideramos que era más oportuno iniciar nuestro trabajo. Este área está comprendida entre Mejorada del Campo al norte y Arganda del Rey-San Martín de la Vega al sur. Aquí la explotación de áridos ha reactivado antiguas explotaciones y abierto otras nuevas, conservándose entre Mejorada y Velilla de San Antonio una amplia secuencia de terrazas (+14-18 m. y +24-25m., +40-41 m., +52-53 m., +82-83 m., +99-100 m., +125-126 m. y +147-148 m., según 


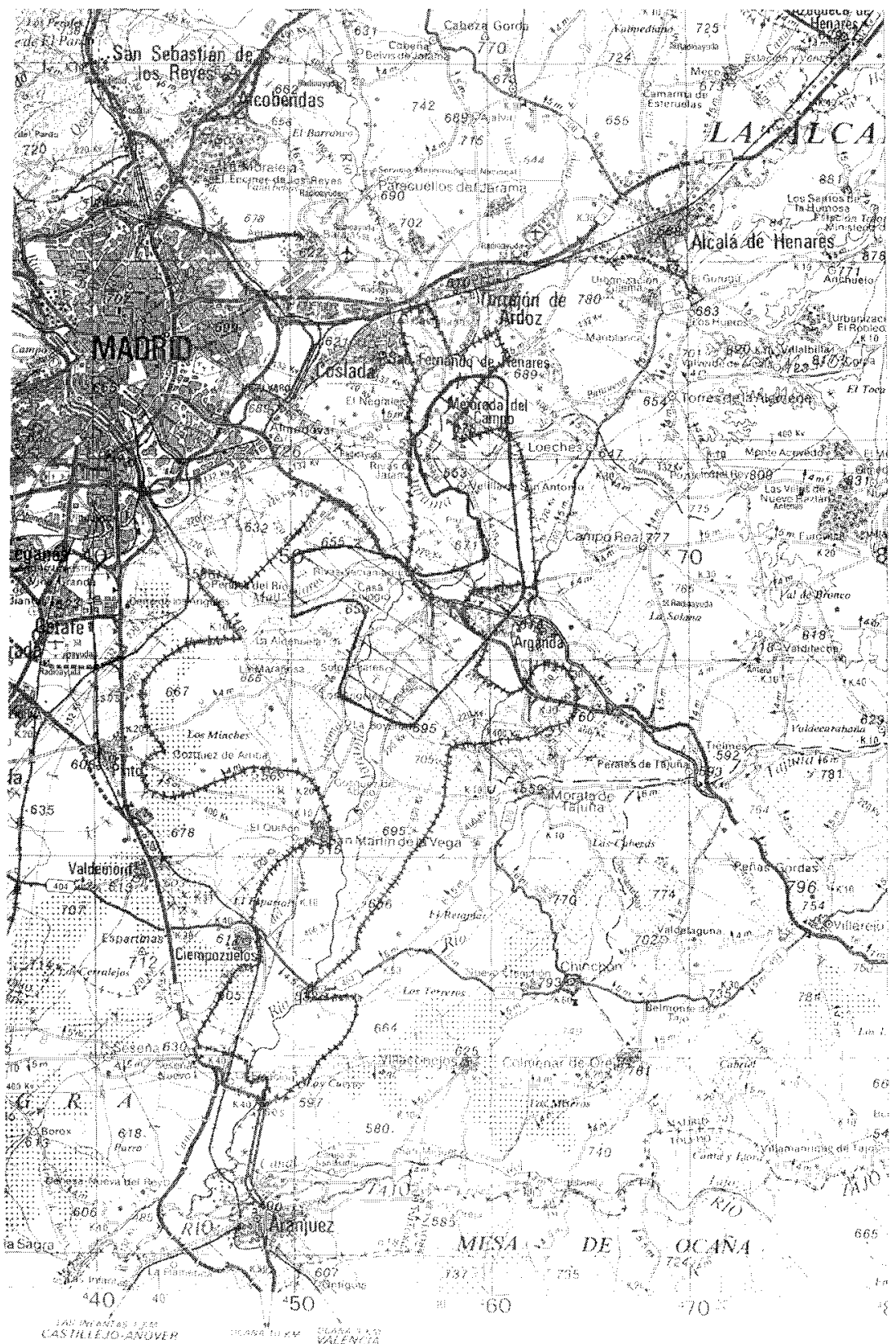

Fig. 2. Mapa del área de estudio. La línea continua señala el área prospectada, la compuesta por cruces los límites del Parque Regional del Sureste. E 1:250000. 
Pérez González en Santonja et alii, 1980), de las que las más altas han sido atribuidas al Pleistoceno inferior. El comportamiento de los depósitos del Jarama, debido al substrato yesífero, ha provocado que las terrazas se hallen hundidas sinsedimentariamente y superpuestas en la terraza de +15 $20 \mathrm{~m}$. (que en este punto presenta dos escalones, $a+17-18 \mathrm{~m} . \mathrm{y}+24-25 \mathrm{~m}$.), convirtiéndola en la más potente, la de estratigrafía más compleja y por tanto la más afectada por la explotación de áridos.

Para elaborar la secuencia estratigráfica de las ocupaciones pleistocenas del Jarama ha sido necesario ubicar conjuntos industriales en las distintas terrazas citadas anteriormente, $y$ en las diferentes unidades litoestratigráficas de la terraza de $+15-20 \mathrm{~m}$. (A. Pérez González ha diferenciado cuatro unidades litoestratigráficas en esta terraza: Arganda I, II, III y IV), que cuenta con abundantes cortes que nos permiten situar la industria lítica estratigráficamente, mientras que en las terrazas situadas topográficamente más altas rara vez se nos ha dado esta circunstancia, por lo que la única actuación posible ha sido la constatación de industria en superficie.

Los materiales arqueológicos encontrados durante el transcurso de las prospecciones son líticos casi en su totalidad salvo el extremo distal de una defensa de Palaeoloxodon antiquus de la que se conservan $82 \mathrm{~cm}$. Si bien, hemos localizado industria en superficie en todas las terrazas, es en las unidades litoestratigráficas de la terraza de $+15-20 \mathrm{~m}$. donde hemos documentado los conjuntos industriales en estratigrafía. Nuestras primeras impresiones vienen a confirmar que:

En Arganda I, donde la potencia máxima observada es de 7-8 $\mathrm{m}$., las industrias muestran características achelenses con rasgos antiguos y las faunas de vertebrados asociadas contienen $\mathrm{Pa}$ laeoloxodon antiquus. Los cortes prospectados nos han permitido atribuir a esta unidad al menos cinco sitios. La citada defensa está en esta unidad y en la misma posición estratigráfica que el yacimiento de Áridos (Santonja et alii, 1980).

Para Arganda II, cuya potencia oscila entre 8 y $10 \mathrm{~m}$. visibles, las industrias se atribuyen a un Achelense medio comparable al del yacimiento de las Acacias y a los niveles inferiores de San Isidro (Santonja et alii, 1980). Situamos once conjuntos.

En Arganda III, con espesores entre 4 y $5 \mathrm{~m}$., y donde no se había documentado industria lítica en estratigrafía, hemos hallado al menos cuatro puntos. Esta unidad estaría marcando el final del Pleistoceno medio. 
Por último, en Arganda IV que representa los estadios más modernos del Pleistoceno superior e incluso del Holoceno se repite la misma situación descrita para Arganda III en relación a la industria, siendo ahora la primera vez que se documenta industria lítica en estratigrafía, al menos 13 sitios.

Como ya señalamos entre nuestros objetivos damos prioridad a la creación de un marco cronológico válido que permita ordenar diacrónicamente los eventos culturales pleistocenos. La unidad Arganda I cuenta con dataciones fiables gracias a los micromamíferos del yacimiento de Áridos, que la ubican hacia el centro del Pleistoceno medio (Santonja et alii, 1980). El resto de las unidades litoestratigráficas de Arganda o las terrazas superiores, tienen referencias cronológicas muy vagas, limitándose su posición cronológica al Pleistoceno medio, salvo las terrazas situadas topográficamente más altas que lo hacen al Pleistoceno inferior. De este modo resulta relevante datar con métodos eficaces las unidades estratigráficas que lo permitan. Arganda II y III no son aptas para la aplicación de métodos absolutos, a su vez el análisis de micromamíferos tampoco es válido dada su práctica inexistencia al corresponderse con medios fluviales muy tractivos y agresivos. Por el contrario, en Arganda IV sí es posible la aplicación de sistemas que proporcionen fechas absolutas, concretamente luminiscencia, para su base, y Carbono 14 AMS, para sus depósitos más modernos. Si consideramos que sus materiales son posteriores a la formación de la terraza aluvial y que reposa sobre Arganda III, su datación (objetivo fundamental del proyecto que está en curso) nos permitirá precisar el marco cronológico de la terraza de $+15-20 \mathrm{~m}$. que es donde se ubican la práctica totalidad de los yacimientos en estratigrafía en la cuenca del Jarama.

\section{A MODO DE REFLEXIÓN}

A comienzos del siglo XXI el potencial arqueológico y paleontológico que encierra las cuencas de estos ríos debe protegerse e investigarse en un momento de delicado equilibrio entre las explotaciones de graveras, la declaración del Parque Natural del Sureste en las áreas de los ríos Jarama y Manzanares y la construcción de infraestructuras como las autopistas M-45, M-50 y R-3 o el trazado del AVE. Una situación donde el patrimonio arqueológico no debe perder la oportunidad de quedar integrado junto con otros como el ecológico. En este sentido propuestas como la anunciada por el Consejero de Medio Ambiente, Carlos Mayor Oreja (EL MUNDO; 
10 de enero de 2000) en la que declara que se está elaborando un plan de ríos en la región que contaría con una partida anual de 1000 millones de pesetas para la conservación de las cuencas fluviales donde se incluyen la protección de humedales, arroyos de núcleos rurales, restauración de las riberas... vienen a demostrar el interés de las Administraciones y las empresas privadas por proteger el patrimonio ecológico y la obligación que tenemos los arqueólogos de poner en valor los restos del pasado para que sean tenidos en cuenta en este tipo de iniciativas.

Los argumentos esgrimidos en este artículo ya fueron trasladados al III Congreso de Arqueología Peninsular celebrado en Vilareal (Portugal) entre el 21 y 27 de septiembre de 1999, exponiendo la mesa de Paleolítico inferior y medio la necesidad y el interés en potenciar los trabajos arqueológicos en el Jarama-Manzanares, elevando a su vez una moción en este sentido que hizo suya el Congreso y que será publicada en las Actas.

\section{AGRADECIMIENTOS}

A la Dirección General de Patrimonio Cultural y Dirección General de Investigación de la Comunidad de Madrid por las facilidades prestadas para el desarrollo de nuestra investigación. A Iván de Castro Pérez por su inestimable ayuda en nuestras prospecciones. A Sergio Ripoll López, Manuel Santonja Gómez y Alfredo Pérez González por su amistad y por asesorarnos en todo momento.

\section{BIBLIOGRAFIA}

BaEna Preysler, J. (1992): Talleres paleolíticos en el curso final del río Manzanares. Patrimonio Arqueológico del bajo Manzanares. V.A.M.

BAYE, J. du (1893): Contribucion a l'étude du gisement paléolithique de San Isidro. Bull. D'Antrp. de Paris, t. IV, 4. ${ }^{a} \mathrm{~s}$.

Obermaier, H. (1916): El Hombre Fósil. Memoria n ${ }^{\circ} 9$ de la Comisión de Investigaciones Paleontológicas y Prehistóricas. Madrid (2. edición: 1925). 457 páginas.

Paz Graellss, M. de la (1897): Fauna Mastológica Ibérica. Real Academia de Ciencias de Madrid. Madrid.

Pérez De Barradas, J. (1923): Yacimientos paleolíticos del valle del Manzanares y del Jarama. Mem. Junta Sup. Exc. y Ant., 50, Madrid. 34 páginas. Madrid.

Pérez De Barradas, J. y Obermaier, H. (1930): Yacimientos paleolíticos del valle del Jarama. Archivo de Prehistoria Madrileña, I: 29-35. Madrid.

Pérez González, A., (1971): "Estudios de los procesos de hundimiento en el valle del río Jarama y sus terrazas (Nota preliminar). Estudios Geológicos, XXVII, 4: 317-324. Madrid.

Pérez González, A., (1994): "La depresión del Tajo.» En Geomorfología de España, M. GutiérRez EloRzA (ed.): 389-434. Editorial Rueda. Madrid.

Pérez González, A., Portero, J.M., diaz Molina, M., Gallardo, J., Aguilar, M.J., Leal, M.C., LOPEZ, N. y AlBERDi, M. (1990): Mapa Geológico de España, escala 1: 50.000; Alcalá de Henares. Instituto Tecnológico Geominero de España. Madrid.

Prado, C. de (1864): Descripción física y geológica de la provincia de Madrid. Reedición del Colegio de Ingenieros de Caminos, Canales y Puertos (1975): 9-352. 
Querol, M.A. y Martínez Díaz, B. (1996a): La gestión del Patrimonio Arqueológico en España. Alianza Editorial Universidad Textos, 161.438 páginas. Madrid.

QueRol, M.A. y MARTínez Díaz, B. (1996b): «El Patrimonio arqueológico en la normativa internacional». Homenaje al Profesor Manuel Fernández Miranda (M.A. Querol y T Chapa, eds.). Complutum extra n. ${ }^{\circ}$ 6-11: 295-306. Madrid.

Querol, M.A. y MARTínez Díaz, B. (1998): «Paso a paso: el tratamiento de los bienes arqueológicos en las leyes de patrimonio de Valencia y Madrid». Complutum 9: 279-291. Madrid.

Santonja, M., (1980): «Madrid, Parque Natural del Paleolítico. Investigaciones recientes en la zona del Jarama». Revista de Arqueología, 1. 4-13. Madrid.

Santonja, M.; López, N. y Pérez GonzÁlez, A. (1980): Ocupaciones achelenses en el valle del Jarama (Arganda, Madrid). Diputación provincial de Madrid, 351 páginas.

SANTONJA, M. y Pérez-GonzÁLEZ, A. (1997): "Los yacimientos achelenses en terrazas fluviales de la Meseta Central española». Cuaternario lbérico. Asociación Española para el Estudio del Cuaternario, Joaquín RodRíguez VIDAL (ed.): 224-234. Huelva.

SANTONJA, M. y QUEROL, M.A. (1977): "Yacimientos paleolíticos del valle del Jarama". Boletín de la Asociación de Amigos de la Arqueología, 8: 4-9. Madrid.

VERNUIL, E. de y LARTET, L., (1863): «Note sur un sílex taillé trouvé dans le diluvium des environs de Madrid". Bulletin de la Société Géologique de France (second series), 20: 698702. 